\title{
RABDOMIOLISIS Y MIOPATÍA COMO ÚNICAS MANIFESTACIONES DE HIPOTIROIDISMO SEVERO SECUNDARIO A TIROIDITIS DE HASHIMOTO
}

\author{
Juan P. Brito ${ }^{1,2, a}$, Juan P. Domecq, ${ }^{2,3, b}$, Gabriela Prutsky",3,b, Germán Málaga²,3,c \\ Larry Young ${ }^{4, d}$, Atil Y. Kargi ${ }^{5, a}$
}

\section{RESUMEN}

La tiroiditis de Hashimoto constituye la causa más frecuente de hipotiroidismo en las regiones sin deficiencia de yodo, es más frecuente en mujeres y muchas veces tiene asociación familiar. Los síntomas y signos del hipotiroidismo son sistémicos y dependen de la duración e intensidad de la deficiencia de la hormona tiroidea. Las manifestaciones neuromusculares, son excepcionalmente los únicos signos clínicos. Se presenta el caso de un paciente joven con una miopatía severa con rabdomiolisis como la única manifestación de hipotiroidismo severo debido a tiroiditis de Hashimoto.

Palabras clave: Hipotiroidismo; Enfermedades musculares; Rabdomiólisis; Tiroiditis, Enfermedades neuromusculares; Manifestaciones neuromusculares (fuente: DeCS BIREME).

\section{RHABDOMYOLYSIS AND MYOPATHY AS THE ONLY MANIFESTATIONS OF SEVERE HYPOTHYROIDISM SECONDARY TO HASHIMOTO'S THYROIDITIS}

\begin{abstract}
Hashimoto's thyroiditis is the most frequent cause of hypothyroidism. In the regions with no iodine deficiency, it is more frequent in women and oftentimes has a familial association. The symptoms and signs of hypothyroidism are systemic and depend on the duration and intensity of the thyroid hormone deficiency. Neuromuscular manifestations are seldom the only symptoms and signs present. We present the case of a young patient with severe myopathy, where rhabdomyolysis was the sole manifestation of severe hypothyroidism secondary to Hashimoto's thyroiditis.
\end{abstract}

Key words: Hypothyroidism; Muscular Diseases; Rhabdomyolysis; Thyroiditis; Neuromuscular diseases; Neuromuscular manifestations (source: MeSH NLM).

\section{INTRODUCCIÓN}

La tiroiditis de Hashimoto es una enfermedad autoinmune que lleva a la destrucción de la glándula tiroides, su incidencia fluctúa entre 0,3 a 1,5 casos por cada 1000 personas por año. Esta enfermedad es más frecuente en mujeres entre los 30 y 50 años de edad (1). En los Estados Unidos la prevalencia de la enfermedad fluctúa entre el 4 al $15 \%$ de la población adulta ${ }^{(2)}$. No se hallaron datos de la prevalencia de esta enfermedad en el Perú; pero, en zonas en donde la ingesta de yodo es suficiente, la tiroiditis de Hashimoto es la causa más común de hipotiroidismo ${ }^{(3)}$.

El cuadro clínico, aunque altamente variable, consta principalmente de bocio indoloro difuso, con manifestaciones sistémicas típicas (3). En oposición, algunos pacientes debutan con un cuadro de tiroiditis subaguda asociada a crecimiento doloroso de la tiroides, estado de tirotoxicosis con manifestaciones sistémicas de enfermedad inflamatoria, y elevada

\footnotetext{
Division of Endocrinology, Diabetes, Metabolism, and Nutrition, Mayo Clinic. Rochester, EE. UU.

Knowledge and Evaluation Research Unit, Mayo Clinic. Rochester, EE. UU.

Unidad de Conocimiento y Evidencia, Universidad Peruana Cayetano Heredia. Lima, Perú.

Department of Rheumatology, Miami Veterans Affairs Medical Center. Miami, EE. UU.

Department of Endocrinology, University of Miami/Jackson Memorial Hospital. Miami, EE. UU.

Médico endocrinólogo, ${ }^{\mathrm{b}}$ médico cirujano, ${ }^{\mathrm{c}}$ médico internista, ${ }^{\mathrm{d}}$ médico reumatólogo

Recibido: 06-11-12 Aprobado: 23-01-13
}

Citar como: Brito JP, Domecq JP, Prutsky G, MálagaG, Young L, Kargi AY. Rabdomiolisis y miopatía como únicas manifestaciones de hipotiroidismo severo secundario a tiroiditis de Hashimoto. Rev Peru Med Exp Salud Publica. 2013;30(1):129-32. 
velocidad de sedimentación globular ${ }^{(4)}$. A pesar de la frecuencia con la que se presentan las manifestaciones neuromusculares en hipotiroidismo (hasta $79 \%$ de pacientes en algunas series) ${ }^{(5)}$, no se cuenta con una explicación fisiopatológica clara y concluyente debido a la complejidad y gran cantidad de funciones celulares y metabólicas afectadas por la falta de la hormona ${ }^{(6)}$.

Las manifestaciones neuromusculares más frecuentes son las mialgias, fatiga, debilidad muscular proximal y reflejos osteotendinosos hipoactivos. En cambio, son infrecuentes los casos severos de rabdomiolisis con niveles marcadamente elevados de creatina fosfoquinasa (CPK) y afección renal ${ }^{(5)} \mathrm{y}$, por lo tanto, potencialmente ignorados. Se reporta el caso de rabdomiolisis y miopatía como únicas manifestaciones de hipotiroidismo severo secundario a tiroiditis de Hashimoto.

\section{REPORTE DE CASO}

Paciente mujer de 24 años de edad natural y procedente de Miami, EE. UU. que acudió al hospital, en esta localidad, con un tiempo de enfermedad de tres semanas de evolución caracterizada por debilidad y rigidez muscular episódica y progresiva. La paciente refirió inicio súbito de los síntomas mientras realizaba ejercicio moderado, presentó bruscamente rigidez muscular lumbar, asociado a dolor intenso (8/10) por debajo de la región patelar, y rigidez simétrica en la misma zona. En los minutos siguientes, la paciente experimentó "entumecimiento" en ambos miembros inferiores, de aproximadamente una hora de duración, que remitió espontáneamente, al igual que el dolor y rigidez lumbar.

En las semanas siguientes, la paciente continuó experimentando estos síntomas de manera episódica y en asociación con la realización de actividad física moderada. Sin embargo, la intensidad de los síntomas fue incrementándose con cada evento. Un día antes del ingreso al hospital la paciente informó ya no poder dar más de cinco pasos seguidos sin fatigarse. Negó debilidad en extremidades superiores o cintura escapular, fasciculaciones, intolerancia al frio, pérdida de cabello, constipación, fiebre, disnea o alteración en el peso. Además, negó haber tomado medicación ni tuvo contacto con personas con síntomas similares. Antecedentes: tiroiditis de Hashimoto en la madre. Antecedentes médicos, quirúrgicos, ginecoobstétricos y sociales no contributorios.

En el examen de ingreso, los signos vitales eran normales. La tiroides era palpable, con características normales y ausencia de dolor. No se halló nada relevante en el examen clínico cardiovascular, pulmonar y abdominal. El examen neurológico evidenció disminución de la fuerza muscular en forma simétrica a predomino de miembros inferiores $(3+/ 5+)$ y en miembros superiores $(4+/ 5+)$, reflejos osteotendinosos disminuidos $(1+/ 4+)$ en forma simétrica, hipoestesia bilateral en los dedos de los pies, además de una disminución de la sensibilidad vibratoria distal a la zona patelar bilateral, dificultad en la realización de la maniobra dedo-talón y alteración en la marcha, arrastrando ambos pies.

En los exámenes auxiliares de ingreso, se constató anormalidad en los valores de creatinina: $1,63 \mathrm{mg} / \mathrm{dL}$ (VN: < 1,2 mg/dL), y CPK: 1103 U/L (VN: < $80 \mathrm{U} / \mathrm{L})$.

La paciente fue admitida con sospecha de compromiso neurológico o muscular. En el plan de trabajo, se consideró la resonancia magnética cerebral, cervical y de médula espinal con contraste; panel inmunológico completo; niveles séricos de vitamina B12; serología para hepatitis; títulos de influenza A y B; perfil tiroideo; anticuerpos contra virus de Epstein Barr; citomegalovirus; sífilis; prueba de HIV; electroforesis de proteínas séricas y urinarias, así como niveles de aldolasa y CPK sérica. Los resultados fueron negativos, exceptuando la aldolasa sérica 9,1 U/L (VN: 1-7,5 U/L); CPK 1959 U/L (VN: 60 $218 \mathrm{U} / \mathrm{L})$ el TSH de $435 \mathrm{mIU} / \mathrm{mL}(\mathrm{VN}: 0,1-4,0 \mathrm{mlU} / \mathrm{mL})$, y la tiroxina 4 libre de 0,04 ng/dL (VN: 0,6-1,8 ng/dL). Debido a estos resultados fueron añadidos al plan de trabajo anticuerpos séricos contra peroxidasa antitiroidea (TPO) y ecografía de la glándula tiroidea. Se encontró una TPO notablemente elevada, $5356 \mathrm{IU} / \mathrm{mL}(\mathrm{VN}:<20 \mathrm{IU} / \mathrm{mL})$ y la ecografía tiroides mostró un crecimiento heterogéneo con vascularidad incrementada. Con la información referida se hizo el diagnóstico de hipotiroidismo por tiroiditis de Hashimoto, complicado con miopatía y rabdomiolisis.

Después de descartar insuficiencia adrenal mediante la prueba de estimulación con cosyntropin, se inició el tratamiento con 100 ug vía oral de levotiroxina. Los síntomas mejoraron progresivamente. La paciente fue dada de alta del hospital cinco días después, presentando solo leve dolor muscular. La reducción de los niveles de CPK fue notable. Se programaron visitas de seguimiento en los servicios de endocrinología y medicina interna. La paciente fue evaluada seis meses después del alta, y manifestó que había reanudado su actividad deportiva y que se mantenía completamente funcional y asintomática. El único tratamiento que recibió fue la sustitución con hormona tiroidea, su nivel de TSH fue de 5,1 $\mathrm{mIU} / \mathrm{mL}$, y el de CPK de $63 \mathrm{U} / \mathrm{L}$.

\section{DISCUSIÓN}

Este caso muestra la importancia del reconocimiento de hipotiroidismo como causa de síntomas 
neuromusculares, los que pueden ser incluso severos y floridos. La tiroiditis de Hashimoto es la principal causa de hipotiroidismo en regiones sin deficiencia de yodo (3), y el musculo esquelético es uno de los tejidos blanco de la hormona tiroidea, por lo que se ve fácilmente afectado por alteraciones de los niveles de la hormona tiroidea. Alrededor del $79 \%$ de los pacientes con hipotiroidismo presentan síntomas musculares, la variedad de estos síntomas dependerá de la severidad y duración del déficit hormonal ${ }^{(5)}$

Dentro de las principales manifestaciones musculares de la enfermedad se encuentran: las paresias de diverso grado, fatigabilidad, dolor, rigidez muscular y calambres, como los presentados por esta paciente. Desafortunadamente la patogénesis de la miopatía inducida por hipotiroidismo no está claramente dilucidada. La propuesta más aceptada plantea que la deficiencia de hormona tiroidea afecta negativamente el metabolismo normal de energía en el músculo, llevando a una producción anómala del glucógeno ${ }^{(7)}$, y la alteración de la cadena oxidativa mitocondrial y del recambio de triglicéridos ${ }^{(8)}$, lo que resulta en un daño muscular directo. Esto se manifiesta como una función muscular inadecuada y dolor, principalmente durante el ejercicio, debido a poca disponibilidad de energía proveniente de la combustión aeróbica de glucógeno. La escasez de vías aeróbicas de energía llevan a la producción de piruvato (vía anaeróbica) con lo que el $\mathrm{pH}$ muscular cae abruptamente produciendo calambres y fatiga al mínimo esfuerzo ${ }^{(9)}$.

La destrucción muscular o rabdomiolisis se asocia a varios meses de deficiencia hormonal. Pocos casos como el presentado son descritos en pacientes con deficiencia aguda o subaguda de hormona tiroidea, siendo la mayoría severos ${ }^{(10)}$. Cabe resaltar que elevaciones no mayores a diez veces el valor normal de CPK y no asociados a rabdomiolisis suelen ser vistos frecuentemente en hipotiroidismo. Esto se debe a alteraciones en la permeabilidad de la membrana celular $\mathrm{y}$, por consiguiente, a una reducción en el aclaramiento de CPK ${ }^{(11)}$.

El hipotiroidismo se asocia también con diferentes alteraciones neurológicas; en diversos estudios electrofisiológicos la incidencia de polineuropatía se encuentra entre 17 a $72 \%{ }^{(5,12)}$. Entre los mecanismos fisiopatológicos más aceptados para explicar estos hallazgos se encuentra el daño y la desmielinización axonal debida a infiltración mucinosa del perineuro y el endoneuro de las neuronas del sistema nervioso periférico ${ }^{(13)}$. Esto provoca mononeuropatías, polineuropatías o neuropatías por atrapamiento, siendo el ejemplo más común el síndrome de túnel del carpo. Además, la reducción del metabolismo de ácidos grasos y carbohidratos conlleva a una menor producción de ATP, esto tendría un rol clave en la degradación de la mielina en los axones neuronales periféricos ${ }^{(14)}$. El diagnóstico de miopatía asociada a hipotiroidismo debe ser mantenido en el diagnóstico diferencial y sospechado de manera especial, en pacientes con debilidad muscular, más aun si estos tienen antecedentes familiares de enfermedad tiroidea ${ }^{(5)}$, como en el presente caso.

La combinación de TSH elevada y de T4 libre baja es suficiente para realizar el diagnóstico ${ }^{(15)}$. En general, las pruebas de electromiografía y biopsia muscular carecen de utilidad en la práctica diaria; son poco utilizadas puesto que son dolorosas, inespecíficas y no conllevan a cambios en el diagnóstico diferencial o el tratamiento de los pacientes ${ }^{(5)}$. La miopatía inducida por hipotiroidismo presenta un pronóstico sumamente favorable una vez iniciada la terapia de reemplazo de hormona tiroidea, los niveles séricos de CPK y TSH suelen normalizarse al cabo de tres o cuatro semanas ${ }^{(16)}$. Sin embargo, la resolución completa de los síntomas puede ser variable y hasta el $30 \%$ de los pacientes presentan síntomas residuales después de un año de controlado el cuadro ${ }^{(5)}$.

Este caso enfatiza la importancia de tener alta sospecha diagnóstica de hipotiroidismo cuando nos enfrentemos a pacientes con miopatías incluso ante la ausencia de los síntomas cardinales de la enfermedad. Esta práctica aseguraría un diagnóstico rápido con la consecuente administración oportuna de tratamiento, como en el caso presentado, previniendo la realización de pruebas diagnósticas invasivas y costosas conllevando a estadías prolongadas.

Contribuciones de autoría: JPBC, JPD, LY y AYK participaron en la concepción y diseño del artículo y recolección de datos. Además, JPBC, JPD y LY realizaron el seguimiento clínico del paciente. Todos los autores participaron en el análisis, redacción del artículo, revisión crítica y aprobación final.

Fuentes de financiamiento: autofinanciado.

Conflictos de interés: los autores declaran no tener conflictos de interés. 


\section{REFERENCIAS BIBLIOGRÁFICAS}

1. Gordin A, Maatela J, Miettinen A, Helenius T, Lamberg BA. Serum thyrotrophin and circulating thyroglobulin and thyroid microsomal antibodies in a Finnish population. Acta Endocrinol (Copenh). 1979;90(1):33-42.

2. Bagchi N, Brown TR, Parish RF. Thyroid dysfunction in adults over age 55 years. A study in an urban US community. Arch Intern Med. 1990;150(4):785-7.

3. Weetman AP, McGregor AM. Autoimmune thyroid disease: further developments in our understanding. Endocr Rev. 1994;15(6):788-830.

4. Kon YC, DeGroot LJ. Painful Hashimoto's thyroiditis as an indication for thyroidectomy: clinical characteristics and outcome in seven patients. J Clin Endocrinol Metab. 2003;88(6):2667-72.

5. Duyff RF, Van den Bosch J, Laman $\mathrm{DM}$, van Loon BJ, Linssen WH. Neuromuscular findings in thyroid dysfunction: a prospective clinical and electrodiagnostic study. J Neurol Neurosurg Psychiatry. 2000;68(6):750-5.

6. Monzani F, Caraccio N, Siciliano G, Manca L, Murri L, Ferrannini E. Clinical and biochemical features of muscle dysfunction in subclinical hypothyroidism. J Clin Endocrinol Metab. 1997;82(10):3315-8.

7. Riggs JE. Acute exertional rhabdomyolysis in hypothyroidism: the result of a reversible defect in glycogenolysis? Mil Med. 1990;155(4):171-2.

8. Khaleeli AA, Griffith DG, Edwards RH. The clinical presentation of hypothyroid myopathy and its relationship to abnormalities in structure and function of skeletal muscle. Clin Endocrinol (Oxf). 1983;19(3):365-76.

9. George G. Hypothyroidism presenting as puzzling myalgias and cramps in 3 patients. J Clin Rheumatol. 2007;13(5):273-5.

10. Benavides VC, Rivkees SA. Myopathy associated with acute hypothyroidism following radioiodine therapy for graves disease in an adolescent. Int J Pediatr Endocrinol. 2010;2010. Epub 2010 Sep 14.

11. Doran GR. Serum enzyme disturbances in thyrotoxicosis and myxoedema. J R Soc Med. 1978;71(3):189-94.

12. Beghi E, Delodovici ML, Bogliun G, Crespi V, Paleari F, Gamba P, et al. Hypothyroidism and polyneuropathy.
J Neurol Neurosurg Psychiatry. 1989;52(12):1420-3.

13. Dyck PJ, Lambert EH. Polyneuropathy associated with hypothyroidism. J Neuropathol Exp Neurol. 1970;29(4):631-58.

14. Argov Z, Renshaw PF, Boden B, Winokur A, Bank WJ. Effects of thyroid hormones on skeletal muscle bioenergetics. In vivo phosphorus-31 magnetic resonance spectroscopy study of humans and rats. J Clin Invest. 1988;81(6):1695-701.

15. Li Y, Nishihara E, Kakudo K. Hashimoto's thyroiditis: old concepts and new insights. Curr Opin Rheumatol. 2011;23(1):102-7.

16. Klein I, Mantell P, Parker M, Levey GS. Resolution of abnormal muscle enzyme studies in hypothyroidism. Am J Med Sci. 1980;279(3):159-62.

Correspondencia: Germán Málaga Rodríguez Dirección: Calle Chavin 159. CC Monterrico. Lima 33, Perú. Teléfono: (51) 992768300

Correoelectrónico: german.malaga@upch.pe

\section{Consulte la versión electrónica de la} Revista Peruana de Medicina Experimental y Salud Pública en www.pubmed.gov

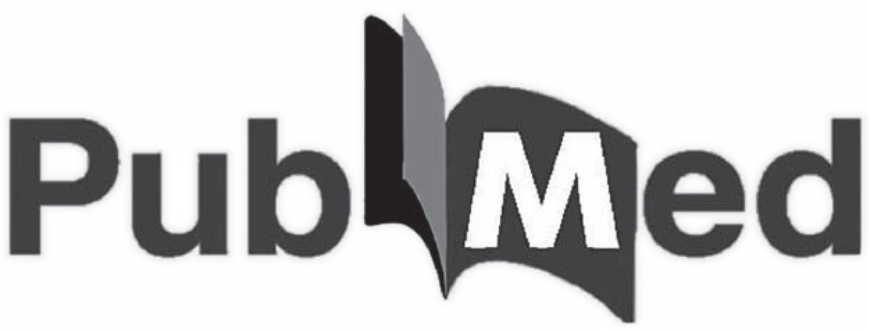

